\title{
Behavior and adrenal activity in a group of zoo golden snub-nosed monkeys, Rhinopithecus roxellana, following social structure change
}

\author{
ZHANG XiaoLi ${ }^{1}$, HE Xin ${ }^{1}$, LIU DingZhen ${ }^{1 *}$, YAO XiuLin ${ }^{1}$, ZHANG YiZhuo ${ }^{2}$, \\ ZHANG EnQuan ${ }^{2}$, ZHANG JinGuo ${ }^{2}$, JIANG GuoHua ${ }^{2} \&$ ZHAO ChangQi ${ }^{1}$ \\ ${ }^{1}$ MOE Key Laboratory of Biodiversity Sciences and Ecological Engineering, Institute of Ecology, Beijing Normal University, \\ Beijing 100875, China; \\ ${ }^{2}$ Beijing Zoo, Beijing 100044, China; \\ ${ }^{3}$ The Analysis and Measurement Centre, Beijing Normal University, Beijing 100875, China
}

Received September 21, 2012; accepted October 30, 2012; published online January 31, 2013

\begin{abstract}
The effects of individual removal by zoo managers on behavior and fecal glucocorticoid changes in snub-nosed monkeys (Rhinopithecus roxellana) were investigated at Beijing Zoo. The results showed that the effect of the removal of three subordinate individuals (DD, male, 4 years; XZZ, male, 3 years and LL, female, 3 years) from a group varied across the remaining group members that may be related to individual's social status, gender and age. The dominant male was impacted less than subordinate individuals in both the frequency and time duration for the four main behavioral categories. The frequency of stereotypic behavior significantly decreased in the dominant female after the removal of three animals $(Z=-2.862, P=0.003)$. Fluctuations were found in both the behavioral frequency and time duration in other remaining individuals. These findings suggest that small, socially stable groups were somewhat resilient to changes in member composition. Moreover, the cortisol level did not change significantly in the remaining members. An alternative method of hormone assay using fecal samples for vulnerable animals was presented, and that may be useful for monitoring wild golden monkeys and other endangered species of animal.
\end{abstract}

animal welfare, artificial separation, resident, golden snub-nosed monkey, Rhinopithecus roxellana, social structure

Citation: Zhang X L, He X, Liu D Z, et al. Behavior and adrenal activity in a group of zoo golden snub-nosed monkeys, Rhinopithecus roxellana, following social structure change. Chin Sci Bull, 2013, 58: 2220-2229, doi: 10.1007/s11434-012-5651-8

For captive animals, anthropogenic agents may lead to changes in group composition. For example, diseased animals may be isolated from the rest of their group by keepers, or animals may be removed for exhibition elsewhere [1-3]. Previous studies on the effects of group change show that the effects of individual removal on zoo animals vary greatly according to species, social context and individuals [4-6]. These differing results raise interesting questions about whether the social dynamics and behavior are affected by the decisions of zoo managers. Nonhuman primates are an ideal group to use to investigate these questions as they usually form stable social groups but are often exchanged between zoos and enclosures or taken out temporarily for

*Corresponding author (email: dzliu@bnu.edu.cn) curing disease or wounds. While the behavior of a group of six zoo golden snub-nosed monkeys (Rhinopithecus roxellana) was being monitored for a long-term study, two animals were moved to a different facility and one to enclosure next door. This provided the opportunity to examine the behavior of the group members before and after the removal of the individuals. As far as it can be ascertained, little research has been conducted to investigate the effects of social structure change caused by zookeepers on both the behavior and stress patterns of zoo nonhuman primates, indicated by fecal cortisol concentrations.

The golden snub-nosed monkey is an endangered species that lives in the Qinling Mountains and other mountain ranges of central China [7]. This species has a complex social organization including several one-male-unit or "nested" 
social organization with group sizes ranging from 20 to 600 individuals. The mating system of this monkey is polygynous and promiscuous [8]. The alpha male in each unit is often taken over by a male intruder followed by a social hierarchy reforming $[9,10]$. Temporary and artificial removal of the alpha male for curing wounds and diseases caused the same effects on the social behavior of group members as those in the wild [11]. However, little literature is available on changes of group member's behavior, including stereotypical behavior associated with fecal cortisol concentrations of golden snub-nosed monkeys, following a removal of non-dominant individuals instead of the alpha male.

Capitalizing on the unexpected removal of three of the study subjects, this study mainly addressed the following questions: what effect does member removal have on both the remaining and the separated animals in a small, socially stable group of nonhuman primates? How can studies like the current one assist the management practices of zoos, especially when planning translocations between facilities? We hypothesized that the removal of non-dominant individuals from a stable group should cause no behavioral changes on the alpha individual, yet a varied behavioral change across other remaining individuals with different social hierarchy status.

\section{Materials and methods}

\subsection{Animals and change in group structure}

The subjects of this study were six golden snub-nosed monkeys housed at the Beijing Zoo. Information about their gender, age, studbook numbers and pedigree is shown in Figure 1. The animals had been housed together for approximately 1 year in an outdoor exhibition hall $(5 \mathrm{~m} \times 5 \mathrm{~m} \times$ $10 \mathrm{~m}$ ) connected to an indoor cage ( $3 \mathrm{~m} \times 8 \mathrm{~m} \times 6 \mathrm{~m})$ containing small trees, bushes, and an apparatus for enrichment. The monkeys were fed daily at $1000 \mathrm{~h}$ cooked eggs, peanuts, coarse grain, fresh vegetables, bananas and apples. Peanuts and grain were usually placed in a plastic puzzle feeder (6 cm diameter, $50 \mathrm{~cm}$ length) with four holes $(3 \mathrm{~cm}$ diameter). The indoor cage was cleaned once per day at $0830 \mathrm{~h}$ and the outdoor hall was cleaned once per week. Animal use and management protocols followed by the zoo met the Chinese Regulations and Standards for Captive Animals.

Two subordinate monkeys (LL and XZZ) were removed and transferred to a zoo quarantine holding facility on 23 May, 2007 prior to their international transportation to the Everland Resort in Korea. A family of three monkeys comprising a male (DZZ), female (YY) and offspring (DIAN) remained in the original enclosure. Another subordinate male (DD) was also separated from the original group of six and housed temporarily with a Guizhou snub-nosed monkey (Rhinopithecus brelichi) (GZ) in a neighboring cage of the same size and environment.

\subsection{Behavioral observations}

Behavioral observations of the whole group were conducted from 22 March to 22 June, 2007 by two observers with prior training in observing golden monkeys. A pilot test revealed differences between observers, so the reliable analysis was run and the methods in classifying behaviors were corrected and standardized until inter-observer correlation was 0.85 or higher [12]. Focal animal sampling and continuous recording were used during the observation process [13]. The monkeys usually woke up at approximately $0700 \mathrm{~h}$ and were most active until their first feeding at $1000 \mathrm{~h}$. For this reason, behavioral observations of the animals in the outdoor enclosure were conducted from 0800-1000 h. Prior to the removal of the two monkeys, observations were conducted for a maximum of $30 \mathrm{~min}$ in intervals of 1-2 d; after the removal, observations were conducted on the four remaining individuals every day except Saturdays or Sundays due to disturbance by zoo visitors.

Two observers conducted observations prior to the removal,

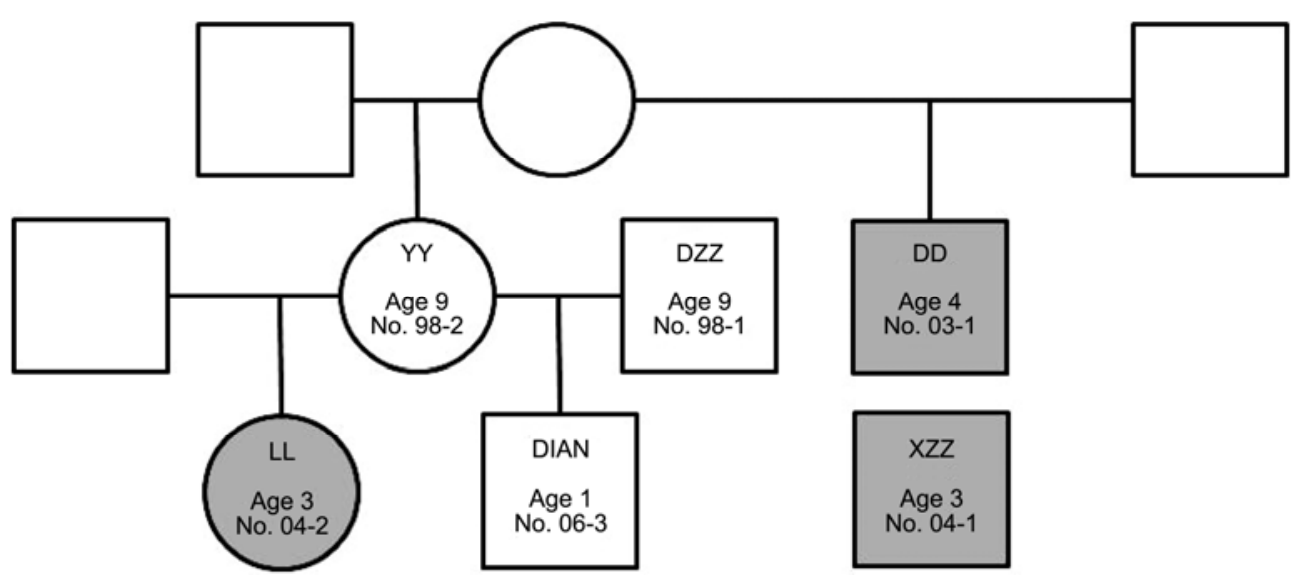

Figure 1 Colony of six golden snub-nosed monkeys. LL and XZZ were transported abroad and DD was placed in a neighboring enclosure. Males are represented by squares and females by circles. Age is years; "No." refers to studbook number. 
one of which continued conducting observations following the removal. Each observer randomly selected a different focal monkey from those present in the outdoor section of the enclosure. The entire group (DZZ, YY, DIAN, DD, $\mathrm{XZZ,} \mathrm{LL)} \mathrm{was} \mathrm{observed} \mathrm{prior} \mathrm{to} \mathrm{member} \mathrm{removal} \mathrm{and} \mathrm{the}$ remaining animals (DZZ, YY, DIAN, DD) were observed following removal.

Four major behaviors (Table 1) were observed and recorded on pre-made check sheets aided by a stopwatch (Citizen DX 9116-A, Shenzhen, Guangdong, China). Digital videos were also taken (SonyDCR-SR85E, Sony, Shanghai, China) at the same time for further behavior check. Behaviors were defined following Ren et al. [9,14,15]. Each observation lasted for about $30 \mathrm{~min}$. Both the frequency and time duration were recorded and used for subsequent statistic analyses. Two other behavioral categories (mating behavior between DZZ and YY, and mother-offspring behavior between YY and DIAN) were also observed and recorded, yet they were so rare during the study that they were excluded from the subsequent analysis.

\subsection{Fecal sample collection}

Fecal samples were collected between 0800-1000 h every day immediately after enclosure cleaning. A fixed time was chosen for collection because cortisol levels in primates fluctuate daily $[16,17]$. Fecal samples were collected from all six animals before and after removal, and were only collected when the producer could be identified; zoo staff helped with this process. We made all our efforts to identify and collect the freshest fecal samples excreted at early in the morning to reduce the sampling error. The freshness of feces was determined by their color and appearance. Usually, fresh feces were in bright dark-grown color with a moist surface. However, fecal samples were not collected enough on the same day on which the behavioral observation was conducted for each individual due to the difficulty in identifying the feces donor and the availability of monkey feces. Thus, fecal samples did not correspond closely to behavioral observation sessions. The total number of fecal samples collected (41 before removal and 52 after removal) ended up being lower than the number of behavioral observations (63 observation blocks before removal and 58 blocks after removal). Fecal samples were sealed in plastic bags and kept in an icebox for approximately 15 min before being transported to the laboratory at Beijing Normal University where they were stored at $-20^{\circ} \mathrm{C}$ until further analysis.

\subsection{Validation, extraction and radioimmunoassay (RIA)}

There is no literature on glucocorticoid (GC) types available for golden monkeys, and physiological and biological validation through the adrenocorticotropic hormone (ACTH) challenge test $[18,19]$ were restricted by the management and animal care practices. Therefore by referring to the available literature on GC types in nonhuman primates [18,19] a high performance liquid chromatography (HPLC) test was conducted with authentic standard compounds of cortisol (Sigma-Aldrich, Shanghai, China) and corticosterone (Sigma-Aldrich, Shanghai, China) so that the GC types in golden monkey feces were identified. HPLC verification was carried out following Wasser et al. [19]. Briefly, two $0.05 \mathrm{~g}$ authentic compounds of cortisol and corticosterone were weighed and dissolved in two identical $80 \%$ methanol solutions (mass-spectrum standard; Galaxy Sky-Grand Technology Co. Ltd., Beijing, China) at fixed volumes of $25 \mathrm{~mL}$. The $80 \%$ methanol solution was made by mixing methanol with double distilled water at a ratio of $8: 2$. Fecal sample extractants were first cleaned by passing through a $0.2-\mu \mathrm{m}$ filter followed by a C-18 matrix column (1200 series; Agilent Technologies, Foster City, California, 94404-1185, USA), and then eluted with $5 \mathrm{~mL}$ of $80 \%$ methanol at a speed of $0.5 \mathrm{~mL} / \mathrm{min}$ [20]. The ultraviolet light detector wave was set to $240 \mathrm{~nm}$. The $80 \%$ methanol was used as the liquid phase. Injections $(10 \mu \mathrm{L})$ were carried out in the following order: standard cortisol, corticosterone solution and extractions of the fecal samples from three golden monkeys (DZZ, XZZ and LL). The extraction was carried out twice for each fecal sample.

Glucocorticoids were extracted from fecal samples following

Table 1 Categories of four major behaviors of snub-nosed monkeys

\begin{tabular}{|c|c|}
\hline Behavior & Definition \\
\hline Stereotypic & $\begin{array}{l}\text { sitting in a dull state without eye-movement for over } 30 \mathrm{~s} \text { at a time; biting the wire mesh or enrichment devices; awaiting food near the } \\
\text { cage gate while hanging on the cage roof; alternating between walking and sitting; licking the cage wall or wire mesh; pacing along } \\
\text { a regular route for over } 30 \mathrm{~s}\end{array}$ \\
\hline Affiliative & $\begin{array}{l}\text { mutual grooming; approaching to another animal within one arm length; following another animal within one arm length; playing with } \\
\text { another animal by pulling on their tail or body; wrestling with another animal; touching for reassurance or as a preliminary action to } \\
\text { grooming; sniffing another animal's face; huddling with another animal mostly while sitting on a platform, and more rarely while } \\
\text { lying down; mutual embrace between two animals, usually father-offspring or mother-offspring }\end{array}$ \\
\hline Submissive & $\begin{array}{l}\text { evading or avoiding another usually dominant or older individual, surrendering by crouching or turning their back towards the superior } \\
\text { individual, or retreating }\end{array}$ \\
\hline
\end{tabular}


the methods of Jurke et al. [21]. Approximately $1.0 \mathrm{~g}$ of feces from each sample was mixed with $3 \mathrm{~mL} \mathrm{H}_{2} \mathrm{O}$ by vortex shaking for $1 \mathrm{~min}$, mixed with $6 \mathrm{~mL}$ ether (Galaxy SkyGrand Technology Co. Ltd., Beijing, China) by vortex shaking for an additional $2 \mathrm{~min}$, vibrated $(150 \mathrm{~g} / \mathrm{min})$ for $2 \mathrm{~h}$ at room temperature, and then centrifuged (1500 g, $15 \mathrm{~min})$. The supernatant fluid was transferred to new test tubes, subjected to evaporation under a stream of nitrogen, dissolved in an assay buffer $(200 \mu \mathrm{L})$, and vibrated $(150 \mathrm{~g} / \mathrm{min})$ overnight. Finally, extractants were transferred to new 500 $\mu \mathrm{L}$ vials and stored at $-20^{\circ} \mathrm{C}$ until radioimmunoassay.

Fecal cortisol levels were measured by radioimmunoassay using a cortisol kit (North China Institute of Biology, Beijing, China), and these data were recorded by a SN-682 radio-immunity gamma-counter (Shanghai Hefu ElectroOptic Corp., Shanghai, China). All samples were measured in a single assay [22]. The correlation efficient for the standard curve was 0.997 , and the intra-assay variation was $12.30 \%(N=96)$. To quantify the dry matter content of the fecal samples, $1 \mathrm{~g}$ of each sample was dried for $8 \mathrm{~h}$ at $120^{\circ} \mathrm{C}$ to constant weight. The dry matter content of each fecal sample was calculated using the following formula:

$$
\alpha=C / G,
$$

where $G$ represents the gross weight of the sample and $C$ the constant weight of the sample. An index of $\beta=1 / \alpha$ was used as a correction factor to calculate the cortisol concentration in dry matter from the concentration assayed in wet fecal samples [23].

\subsection{Data analysis}

The time spent displaying each behavior was calculated as a proportion of the total observation time in each observation window (30 min) for analysis. For the frequency occurrence of each behavior, it was also standardized to data value of per 30-min session. In considering the highly different behavioral changes for individuals with different social status, and by referring to the methods employed by Lindburg et al. [24], Wilson et al. [25] and Moreira et al. [26] for small sample sizes, the behavioral changes of four main behavioral categories over observation days for each individual were first descriptively reported. The Mann-Whitney U test was used to compare the occurrence of different behaviors (both in frequency and proportion of time) before and after the removal individually instead of pooling the data across individuals or comparing them as a group. For the cortisol changes, data collected from each animal before and after the removal was compared on average by each individual using the Mann-Whitney U test. The alpha was 0.05 and all tests were two-tailed. For the behavioral comparison of each individual, we used a Bonferroni corrected $P$-value of 0.01 to designate a significant result, and also reported findings with $0.01<P<0.05$ as trends [25]. Last, the relationships and social interaction changes between individuals of the whole group were also descriptively reported using socio-grams before and after the removal [4].

\section{Results}

\subsection{Individual changes in behavior}

The behavioral changes caused by animal removal varied greatly with each animal's social status, age and gender. For the alpha male, DZZ, the frequency and percentage of time of his aggressive, stereotypic and affiliative behaviors were less affected by the removal (Figure 2a,b,d), submissive behavior was not observed in this male (Figure 2c). For the dominant female, YY, the frequency of her stereotypic behavior was significantly lower after the removal $(Z=-2.862$, $P=0.003$; before: $15.00 \pm 1.543$, after: $7.00 \pm 0.548$ ) (Figure $3 \mathrm{~d})$. The frequencies and percentages of her other behaviors did not change significantly (Figure 3a,b,c). For the juvenile male, DIAN, the frequency of his affiliative behavior showed a trend of declining after removal $(Z=-2.325 ; P=0.019$; before: $19.00 \pm 1.922$, after: $10.00 \pm 2.608$, Figure $4 \mathrm{~b}$ ). The frequency and percentage of his other behaviors did not change significantly (Figure 4a,c,d). For the subordinate male, DD, the frequency of his aggressive behavior showed a trend of decline $(Z=-2.146, P=0.028$; before: $5.38 \pm 0.844$, after: $2.00 \pm 0.707$ ) (Figure 5a). The percentage of his submissive behavior completely disappeared $(\mathrm{Z}=-2.766, P=$ 0.004 ; before: $0.429 \pm 0.016$, after: $0.0266 \pm 0.00832$ ) after he was housed with a Guizhou snub-nosed monkey next door to his original group (Figure 5c).

Moreover, the relationship and interactions between individuals also changed greatly after the removal. The aggressiveness of DZZ to DIAN enhanced after DD, LL and XZZ were removed. DD began to show aggressive behavior to his new cage mate, the Guizhou (GZ) snub-nosed monkey (Figure 6a). DZZ displayed weak affiliative behavior to his son, DIAN in addition to YY after DD, LL and XZZ were removed. DD also showed some affiliative behavior to his new cage mate even though GZ is not the same subspecies (Figure 6b). DIAN showed weak submissive behavior to his father, and no submissive behavior was found between DD and his new cage mate GZ.

\subsection{HPLC analysis and hormonal changes}

The retention time of authentic cortisol was $4.403 \mathrm{~min}$, and that of corticosterone was 5.246 min (Figure 7). Extractants from the fecal samples of DZZ, XZZ and LL showed peaks

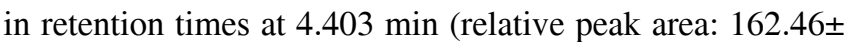
$5.46, n=3$ ) and 5.246 min (relative peak area 88.69 \pm 11.04 , $n=3$ ). The average ratio of the relative peak area of cortisol to corticosterone was $2.03: 1$. Thus, cortisol is the main glucocorticoids hormone in the golden monkey. 

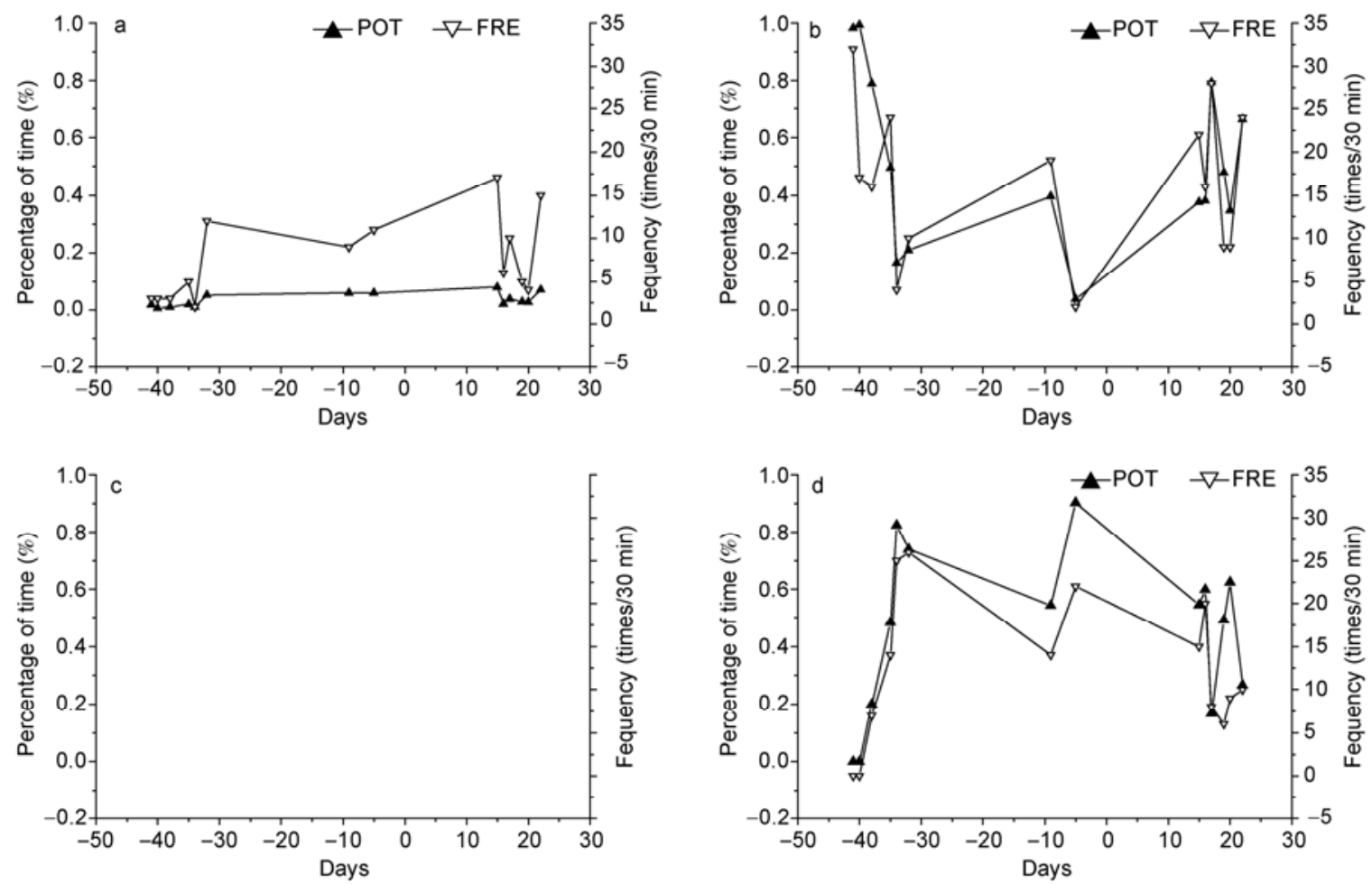

Figure 2 Percentage and frequency of agnostic, affiliative, submissive and stereotypic behaviors preceding and following group changes in DZZ. a, Agnostic behavior; $\mathrm{b}$, affiliative behavior; $\mathrm{c}$, submissive behavior (not observed in this individual); $\mathrm{d}$, stereotypic behavior. The numbers under the $x$ axis indicate the days of observation before (negative) or after (positive) the individual removal. POT, Percentage of time; FRE, frequency (the same below).
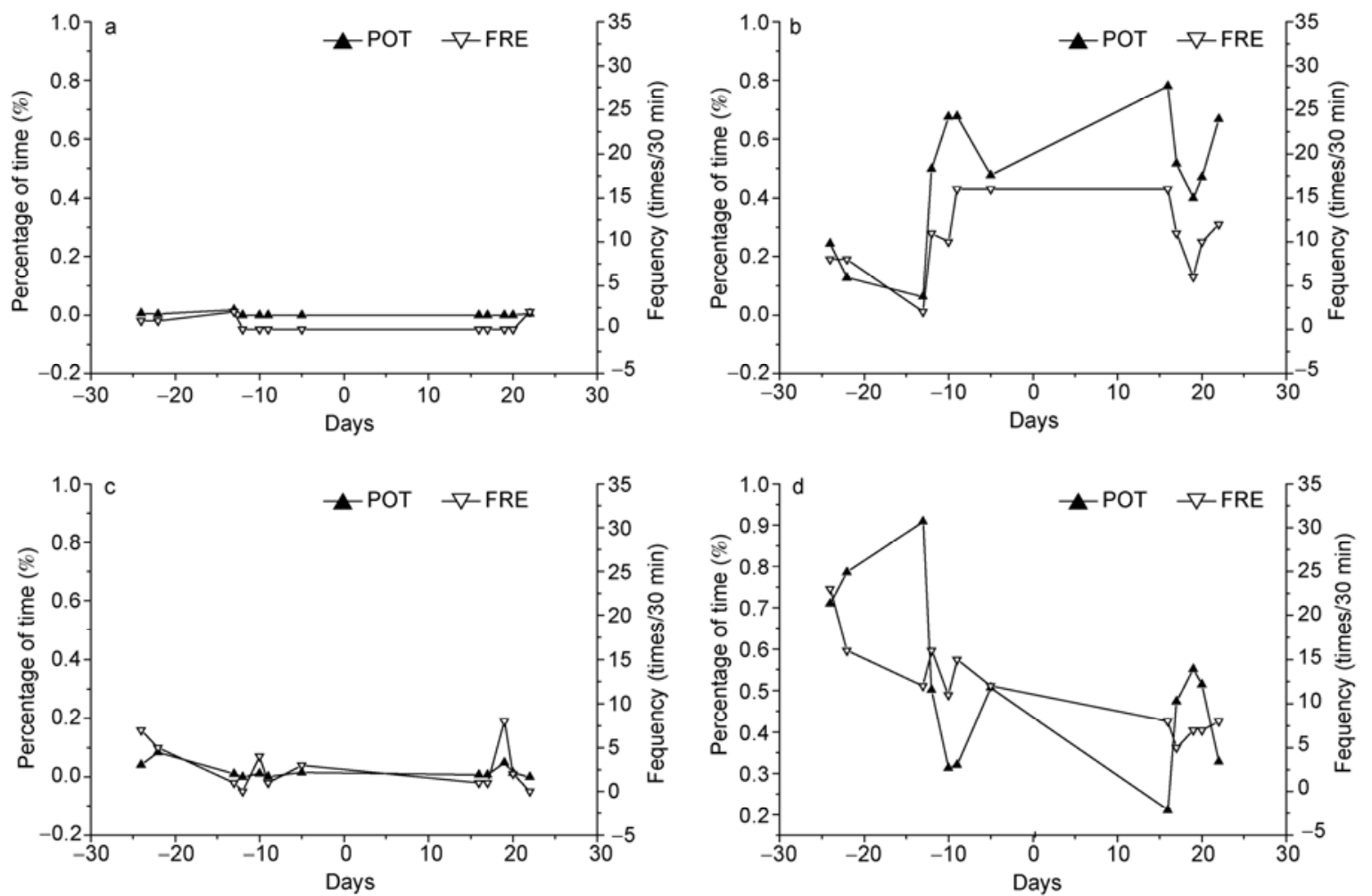

Figure 3 Percentage and frequency of agnostic, affiliative, submissive and stereotypic behaviors preceding and following group changes in YY. a, Agnostic behavior; b, affiliative behavior; c, submissive behavior; $\mathrm{d}$, stereotypic behavior. 

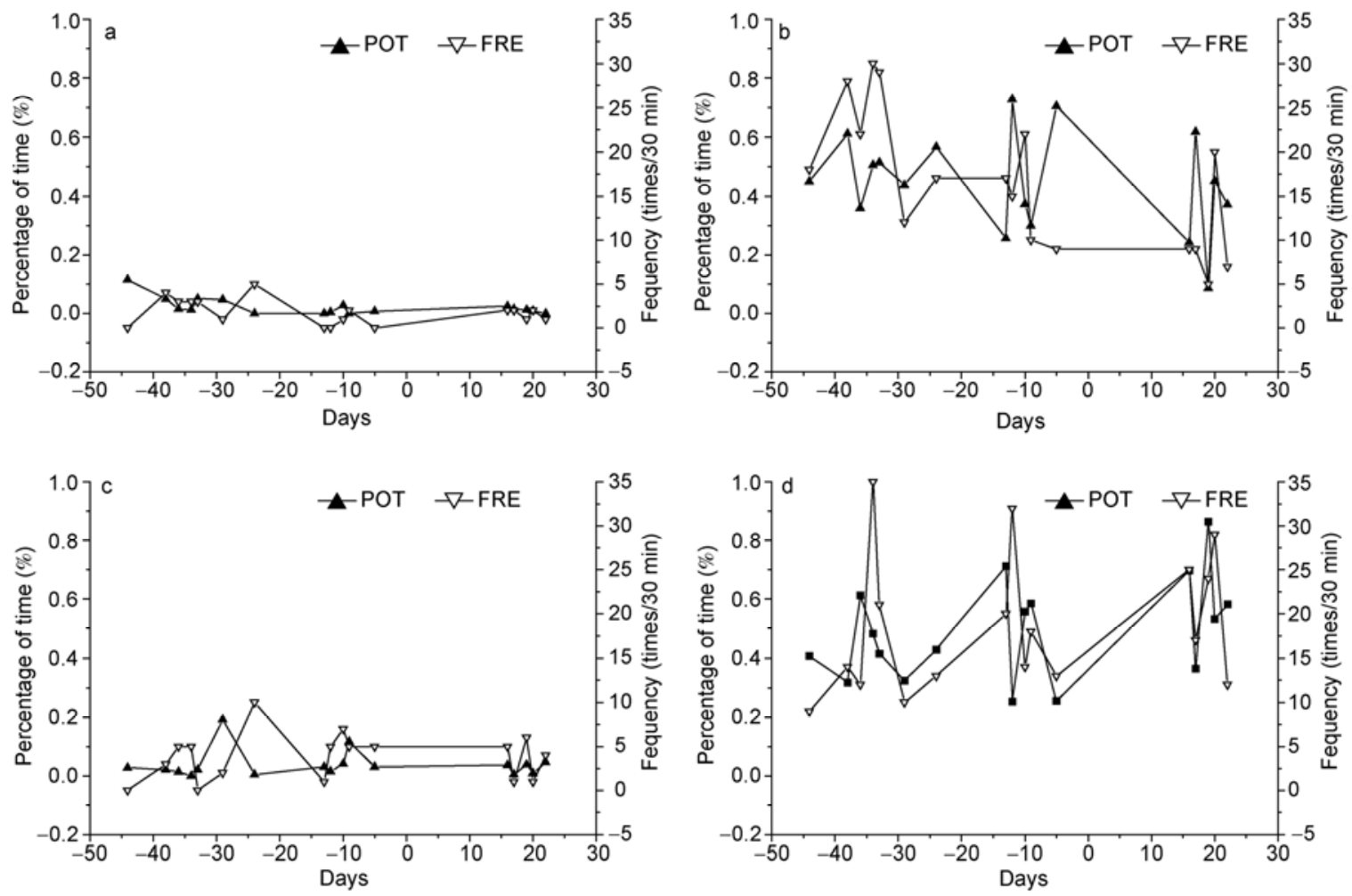

Figure 4 Percentage and frequency of agnostic, affiliative, submissive and stereotypic behaviors preceding and following group changes in DIAN. a, Agnostic behavior; b, affiliative behavior; c, submissive behavior; d, stereotypic behavior.
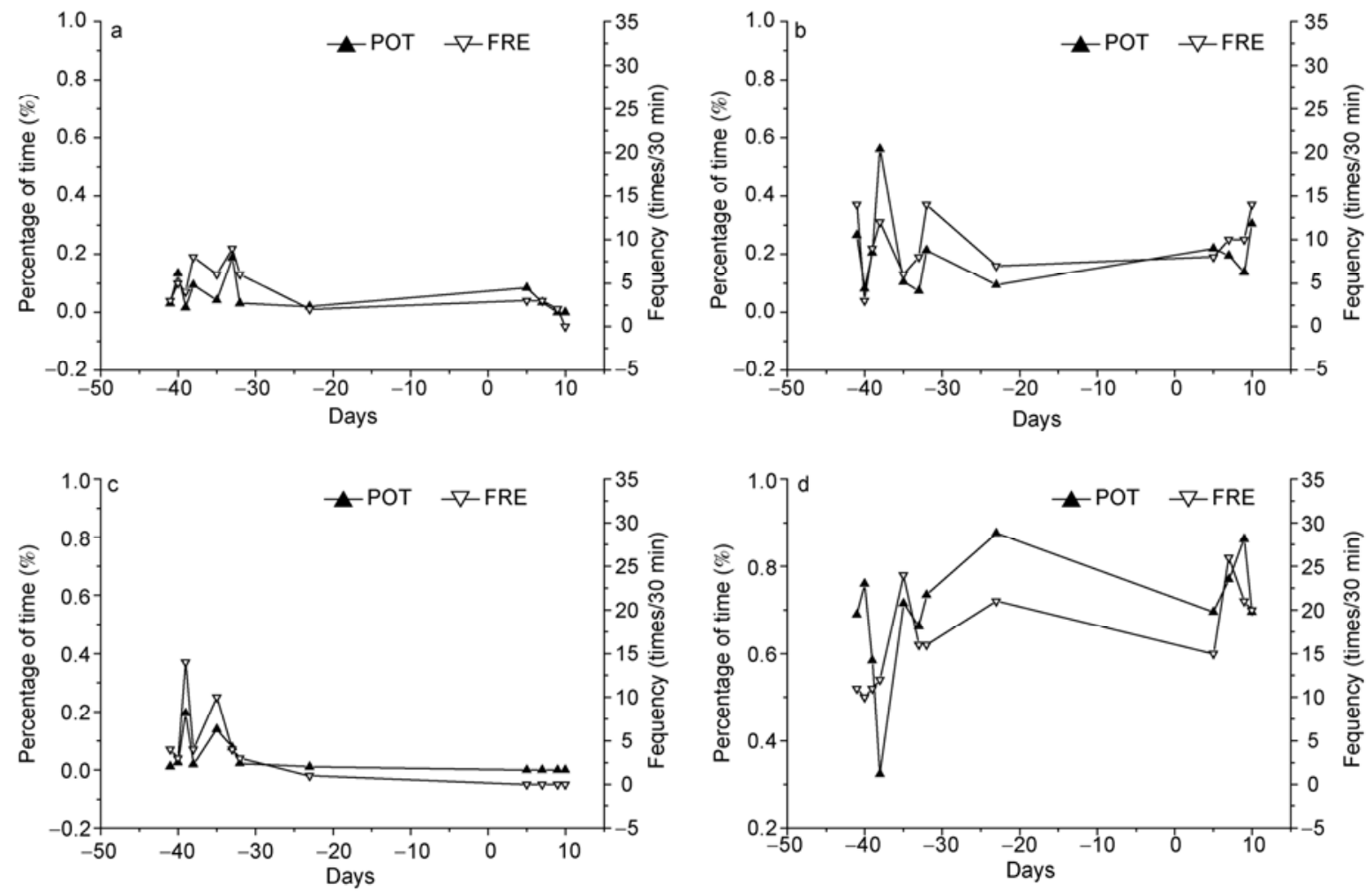

Figure 5 Percentage and frequency of agnostic, affiliative, submissive and stereotypic behaviors preceding and following group changes in DD. a, Agnostic behavior; b, affiliative behavior; c, submissive behavior; d, stereotypic behavior. 

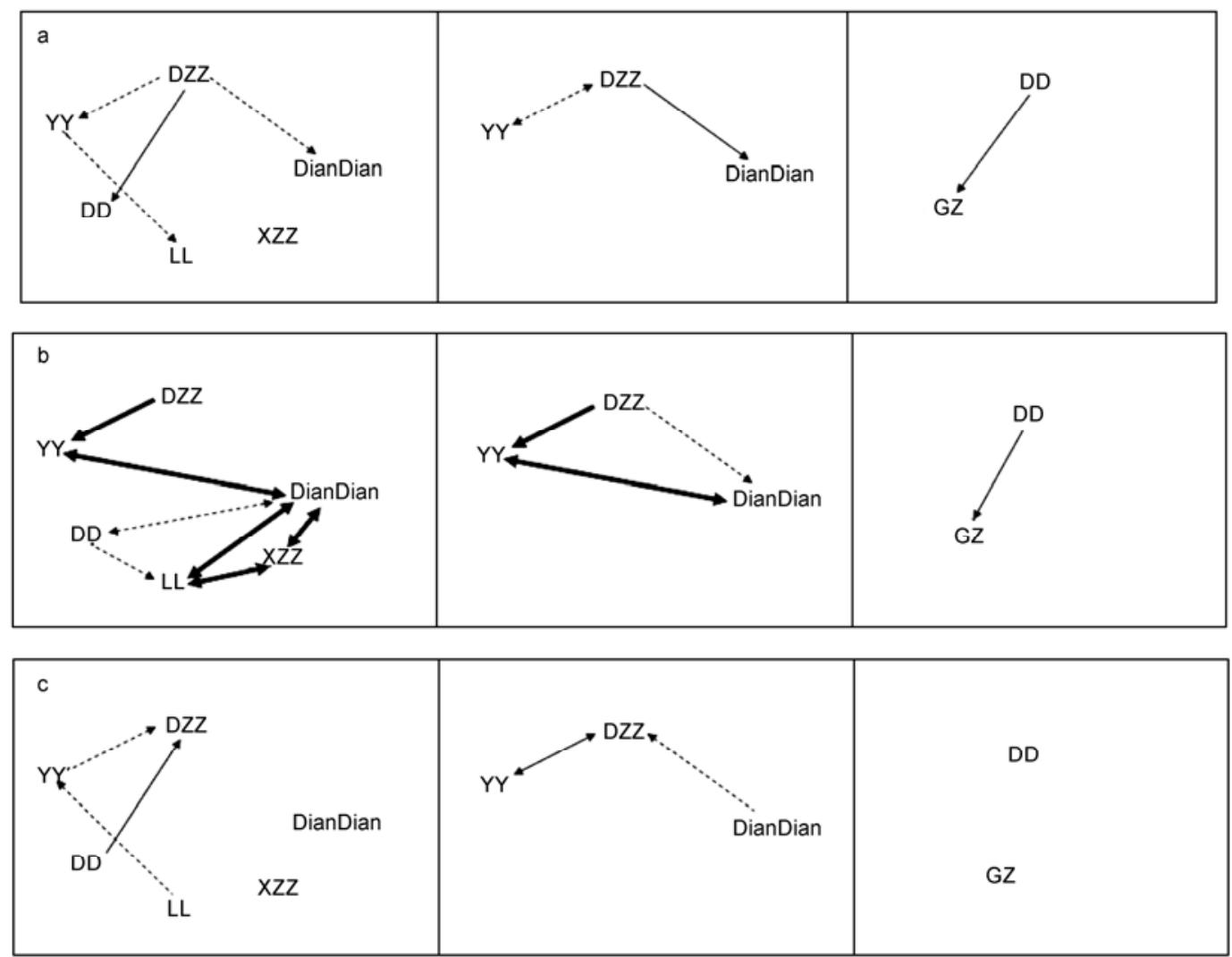

Figure 6 Relationships amongst the group before and after group change. Connections indicate transmission of (a) agnostic, (b) affiliative and (c) submissive behavior. Before the removal all animals were zoo in one cage. After removal, XZZ, LL and DD were moved out of the cage. DD was moved to a cage next door kept a Guizhou snub-nosed monkey (GZ). The first panel (left) means the relationship before removing. The second (middle) and third (right) panels mean the relationship between members of group after removing. A broken line means little interaction between animals. A thin line means moderate levels of exchange between animals. A thick line means a significant amount of interaction between animals.

After the removal, the fecal cortisol levels increased for all monkeys except DD; however, only XZZ's fecal cortisol level significantly increased $(Z=-2.229, P=0.025$; before: $34.065 \pm 11.047$, after: $52.871 \pm 5.622$, Figure 8 ). No similar result was found in other monkeys. For DD, his fecal cortisol level significantly declined after the removal $(Z=-2.089$, $P=0.025$; before: $55.207 \pm 7.200$, after: $7.155 \pm 3.925$, Figure 8 ).

\section{Discussion}

Removing three subordinates from a six-member group of zoo snub-nosed monkeys did not significantly affect behavioral profiles, time spent engaged in key behaviors, or fecal cortisol levels among those animals that remained. Nevertheless, it did bring about slight changes in the frequency of behavior, as well as changing relationships between group members. This is an interesting finding helpful to the management of primates that tend to form close social bonds with each other under captive and field conditions [27,28]. Group members remaining in the enclosure did not demonstrate signs of stress, protest or despair [4,6], suggesting that they had little attachment to the monkeys removed from the enclosure within 1 year cohabitation [5]. The animals removed from the group consisted of a subordinate female (daughter of the dominant female), a male (unrelated to the other animals) and a sub-adult male (coefficient of kinship with the dominant female is $1 / 4)$. The position of the first two individuals in the social hierarchy, gender and age made them ideal subjects for transferring to another facility. In addition, the concurrent removal of the subordinate yet reproductively mature male, DD, to an adjacent enclosure, actually appeared to restore group dynamics by lowering levels of aggression within the remaining member of the group. The results of this study support the idea that the management of captive animals, especially those actions that change group composition, need to reflect an understanding of social processes and group dynamics [3].

At the individual level, changes in behavior were observed. Limitations in the scope of this study and small sample size may have contributed to a lack of statistical significance throughout these data. A larger sample size, prolonged observations [4] and a consideration of longerterm change [5] should be incorporated into future studies. However, a discussion of the trends observed while acknowledging the statistical caveats will be of value to the 

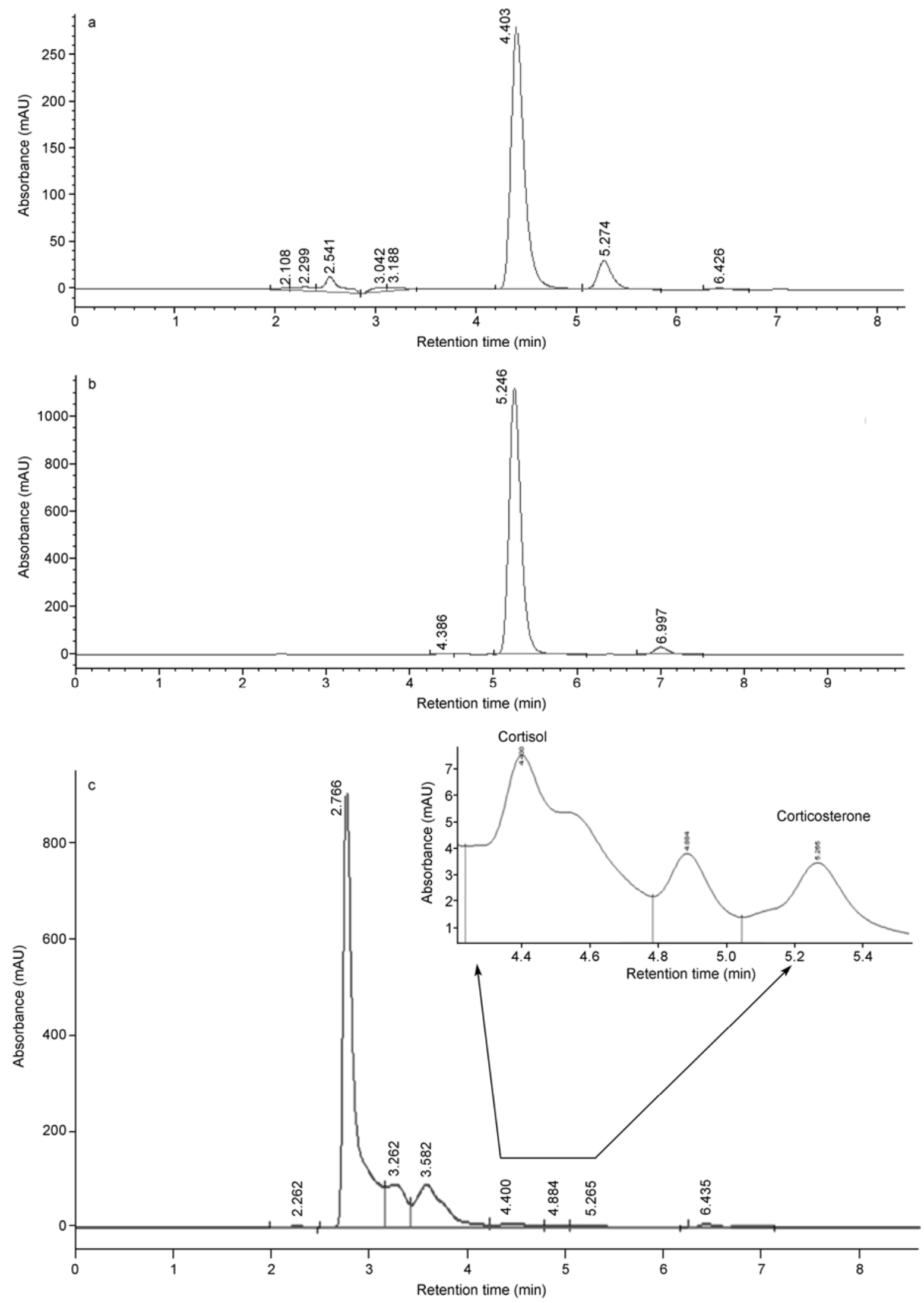

Figure 7 The HPLC spectrograms of authentic chemicals of cortisol (a) at retention time $=4.40 \mathrm{~min}$ and corticosterone (b) at retention time $=5.246 \mathrm{~min}$ and fecal sample of DZZ (c). The inset figure on Figure 7c shows the enlarged two peaks of cortisol (retention time $4.40 \mathrm{~min}$ ) and corticosterone (retention time at around $5.246 \mathrm{~min})$. 


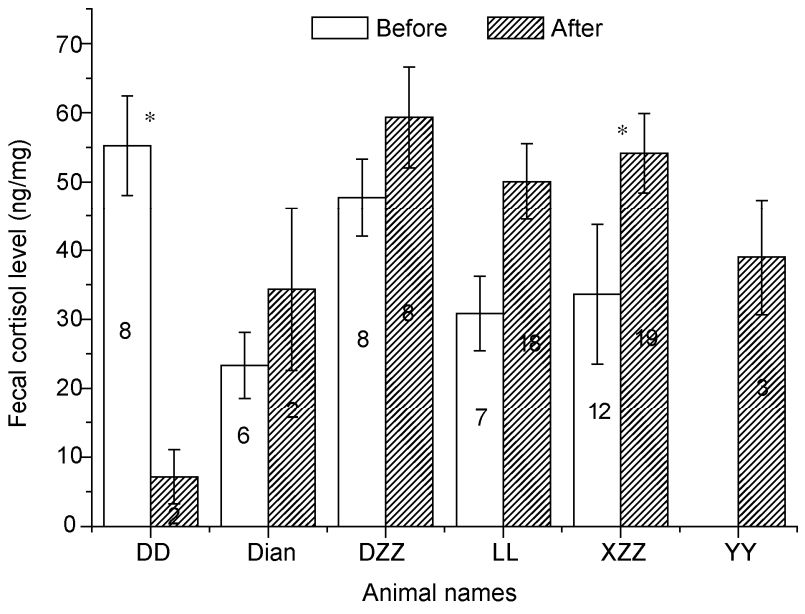

Figure 8 Fecal cortisol levels before and after removal. *Significance at $P<0.05$. There was no fecal cortisol measurement for $\mathrm{YY}$ before the change. Numbers inside the bars indicate the fecal sample size for each animal.

understanding of the effect of this change on individual captive primates.

Changes in behavior are associated with an individual's social status and adaptation to a new group or environment $[29,30]$. The dominant adult male's (DZZ) behavior was not significantly affected by the change in group composition, because DZZ's status was not changed and challenged after the removal (Figure 6). YY (the dominant female) showed less stereotypic behavior because of less aggression and more submissive behavior from DZZ (Figure 6a,c). After the removal of LL, YY was the only adult female in the group, and this alleviated pressure on YY from LL and other individuals (Figure 6a,c). This change may result in her less stereotypic behavior. DIAN showed less affiliative behavior because he lost the only peers willing to play with him (DD, XZZ and LL). After XZZ, LL and DD were removed from the group, none of the remaining monkeys engaged in play with DIAN. Thus, he showed less affiliative behavior due to loss of his peers (Figure 4b). DD's removal and cohabitation with another species of monkey decreased his submissive behavior. DD was clearly threatened by DZZ before the change in group membership. DD showed equally agonistic and affiliative behavior to his new cage mate (Figure 6a,b,c). DD's submissive behavior disappeared but stereotypic behavior rose, and then declined in the new cage (Figure 5d). These changes in behavior occurred because they adapted to their new social environments [31,32]. As to the remaining individuals, the frequency and percentage of time engaged in these behaviors both changed. This change may be related to despair at the loss of their peers LL and XZZ, and in DIAN and DD's case, in the loss of each other [5], as they frequently played together before separation (data not shown). Adverse early experiences such as separation can compromise behavioral and psychological adjustment in primates, and can lead to stress-related behavioral deficiencies [33,34].

Fecal cortisol levels and submissive behavior declined in DD following his separation and cohabitation with another species. In the former social environment, DD's access to food and females (YY) was restricted by DZZ. Aggression in adult animals directed towards same-sex group members in primates is normally triggered by competition for limited resources such as females or food [35]. In wild golden snubnosed monkey groups, the resident adult male or highestranking individual initiates most aggressive behavior $[10,36,37]$. The abnormal situation of another reproductively mature adult male in close habitation with a one-male unit placed DD under stress. Therefore, the abrupt reduction in submissive behavior and fecal cortisol levels in DD resulted from his separation from the dominant male DZZ. XZZ and LL moved out from the whole group. They were stressed by short distance transportation and a totally new environment. XZZ's change in cortisol level is statistically significant. Subordinate animals appear to behave shyly when responding to a novel environment or stimulus [30]. So XZZ's increase in cortisol level might be resulted from the new environment.

Last, this study employed a novel method to quantify glucocorticoid compounds in feces because zoo management practices prohibited the collection of blood. In hormonal studies of endangered and rare species, it is often difficult to follow the standard protocol for stress-related hormone assay by ACTH challenge test and validation analysis $[18,19,38]$. Thus, authentic compounds of two major glucocorticoids were used to verify the main compounds via an alternative method, HPLC. Despite unavoidable limitations, this method was used to quantify main compounds in the feces of the golden snub-nosed monkeys from the ratio of the concentration of both major glucocorticoid compounds. The method in this study has shown that the main glucocorticoid in golden snub-nosed monkeys is cortisol and can be applied to wider conservation research on other rare and endangered species.

\section{Conclusions}

According to this study, removing subordinate individuals from a group of captive golden monkeys may not always negatively affect the welfare of the remaining animals. However, caution is still advised in extending the current results to broad captive golden monkey management. As is clear from this study, the dominant male's behavior and fecal cortisol level did not change statistically. YY performed less stereotypic behavior after the subordinate members being moved out. It was also found that the juvenile DIAN performed less bouts of affiliative behavior after losing his peers, XZZ, LL and DD. The sub-adult DD's fecal cortisol level and submissive behavior decreased, while XZZ's cortisol increased. Thus, the final effects of 
individual monkey removal from a group greatly depend on particular individuals, and relationships between individuals.

The authors would like to thank the staff at Beijing Zoo for their help in fecal sample collection and to Professor LI Baoguo for his helpful suggestions during this project. This work was supported by Beijing Natural Science Foundation (5082011), the National Natural Science Foundation of China (30470233) and Centre of Analysis \& Measurement of Beijing Normal University.

1 Bush M. Methods of capture, handling, and anesthesia. In: Kleiman D G, Allen M E, Thompson K V, eds. Wild Mammals in Captivity. Chicago, USA: The University of Chicago Press, 1996. 25-40

2 Hinshaw K C, Amand W B, Tinkelman C L. Preventive medicine. In: Kleiman D G, Allen M E, Thompson K V, et al., eds. Wild Mammals in Captivity. Chicago: The University of Chicago Press, 1996. 16-24

3 Watts E, Meder A. Introduction and socialization techniques for primates. In: Kleiman D G, Allen M E, Thompson K V, et al., eds. Wild Mammals in Captivity. Chicago: The University of Chicago Press, 1996. 67-77

4 Lemasson A, Gautier J P, Hausberger M. A brief note on the effects of the removal of individuals on social behaviour in a captive group of campbell's monkeys (Cercopithecus campbelli campbelli): A case study. Appl Anim Behav Sci, 2005, 91: 289-296

5 Tarou L R, Bashaw M J, Maple T L. Social attachment in giraffe: Response to social separation. Zoo Biol, 2000, 19: 41-51

6 Burman O, Owen D, AbouIsmail U, et al. Removing individual rats affects indicators of welfare in the remaining group members. Physiol Behav, 2008, 93: 89-96

7 Zhang Y Z, Wang S, Quan G Q. On the geographical distribution of primates in China. J Hum Evol, 1981, 10: 215-226

8 Zhang P, Watanabe K, Li B, et al. Social organization of Sichuan snub-nosed monkeys (Rhinopithecus roxellana) in the Qinling Mountains, Central China. Primates, 2006, 47: 374-382

9 Ren R M, Su Y J, Yan K H, et al. Social relationships among golden monkeys in breeding cages. Acta Psychol Sin, 1990, 22: 277-282

10 Li B G, Li H Q, Zhao D P, et al. Study on dominance hierarchy of the Sichuan snub-nosed monkey (Rhinopithecus roxellana) in Qinling Mountains. Acta Theriol Sin, 2006, 26: 18-25

11 Ren B P, Liang B, Zhang S Y, et al. Effects of temporary removal and replacement of the apha male on social behavior of the captive Sichuan snub-nosed monkey Rhinopithecus roxellanae. Acta Zool Sin, 2007, 53: 755-762

12 Lehner P N. Handbook of Ethological Methods, 2nd ed. Cambridge: Cambridge University Press, 1996. 671

13 Martin P, Bateson P. Measuring Behaviour. Cambridge: Cambridge University Press, 1986. 200

14 Ren B P, Xia S Z, Li Q F, et al. Male copulatory patterns in captive Sichuan snub-nosed monkey (Rhinopithecus roxellana). Acta Zool Sin, 2002, 48: 577-584

15 Ren R M, Yan K H, Su Y J, et al. The Society of Golden Monkeys. Beijing: Peking University Press, 2000. 222

16 Raminelli J L F, de Sousa M B C, Cunha M S, et al. Morning and afternoon patterns of fecal cortisol excretion among reproductive and non-reproductive male and female common marmosets, Callithrix jacchus. Biol Rhythm Res, 2001, 32: 159-167

17 Torres-Farfan C, Valenzuela F J, Ebensperger R, et al. Circadian cortisol secretion and circadian adrenal responses to ACTH are maintained in dexamethasone suppressed capuchin monkeys (Cebus apella).
Am J Primatol, 2008, 70: 93-100

18 Touma C, Palme R. Measuring fecal glucocorticoid metabolites in mammals and birds: The importance of validation. Ann N Y Acad Sci, 2005, 1046: 54-74

19 Wasser S K, Hunt K E, Brown J L, et al. A generalized fecal glucocorticoid assay for use in a diverse array of nondomestic mammalian and avian species. Gen Comp Endocrinol, 2000, 120: 260-275

20 Shackleton C H. Profiling steroid hormones and urinary steroids. J Chromatogr, 1986, 379: 91-156

21 Jurke M H, Czekala N M, Lindburg D G, et al. Fecal corticoid metabolite measurement in the cheetah (Acinonyx jubatus). Zoo Biol, 1997, 16: 133-147

22 Engelhardt N K, Groothuis T. Measuring steroid hormones in avian eggs. Ann NY Acad Sci, 2005, 1046: 181-192

23 Li C W, Jiang Z G, Jiang G H, et al. Seasonal changes of reproductive behavior and fecal steroid concentrations in Pere David's deer. Horm Behav, 2001, 40: 518-525

24 Lindburg D G, Czekala N M, Swaisgood R R. Hormonal and behavioral relationships during estrus in the giant panda. Zoo Biol, 2001, 20: $537-543$

25 Wilson M L, Bashaw M J, Fountain K, et al. Nocturnal behavior in a group of female African elephants. Zoo Biol, 2006, 25: 173-186

26 Moreira N, Brown J L, Moraes W, et al. Effect of housing and environmental enrichment on adrenocortical activity, behavior and reproductive cyclicity in the female tigrina (Leopardus tigrinus) and margay (Leopardus wiedii). Zoo Biol, 2007, 26: 441-460

27 Richard A F. Primates in Nature. NY, USA: W. H. Freeman and Company, 1985. 558

28 Mitani J C, Gros-Louis J, Manson J H. Number of males in primate groups: Comparative tests of competing hypotheses. Am J Primatol, 1996, 38: 315-332

29 Darrow P A, Shivik J A. Bold, shy, and persistent: Variable coyote response to light and sound stimuli. Appl Anim Behav Sci, 2009, 116: 82-87

30 Mettler A E, Shivik J A. Dominance and neophobia in coyote (Canis latrans) breeding pairs. Appl Anim Behav Sci, 2007, 102: 85-94

31 Clarke M R, Harrison R M, Didier E S. Behavioral, immunological, and hormonal responses associated with social change in rhesus monkeys (Macaca mulatta). Am J Primatol, 1996, 39: 223-233

32 Franceschini M D, Rubenstein D I, Low B, et al. Fecal glucocorticoid metabolite analysis as an indicator of stress during translocation and acclimation in an endangered large mammal, the Grevy's zebra. Anim Conserv, 2008, 11: 263-269

33 Reimers M, Schwarzenberger F, Preuschoft S. Rehabilitation of research chimpanzees: Stress and coping after long-term isolation. Horm Behav, 2007, 51: 428-435

34 Harlow H, Dodsworth R, Harlow M. Total social Isolation in monkeys. Proc Natl Acad Sci USA, 1965, 54: 90-97

35 Zhang P, Watanabe K, Li B G, et al. Social organization of Sichuan snub-nosed monkeys (Rhinopithecus roxellana) in the Qinling Mountains, Central China. Primates, 2006, 47: 374-382

36 Chen F, Min Z, Luo S, et al. Observation on the behavior and ecology of golden monkeys (Rhinopithecus roxellana) in Qinling Mountains. Acta Theriol Sin, 1983, 3: 141-146

37 Li B G, Zhao D P. Copulation behavior within one-male groups of wild Rhinopithecus roxellana in the Qinling Mountains of China. Primates, 2007, 48: 190-196

38 Palme R, Rettenbacher S, Touma C, et al. Stress hormones in mammals and birds: Comparative aspects regarding metabolism, excretion, and noninvasive measurement in fecal samples. Ann NY Acad Sci, 2005, 1040: 162-171

Open Access This article is distributed under the terms of the Creative Commons Attribution License which permits any use, distribution, and reproduction in any medium, provided the original author(s) and source are credited. 